\title{
Hybrid Small Animal Imaging System Combining Magnetic Resonance Imaging With Fluorescence Tomography Using Single Photon Avalanche Diode Detectors
}

\author{
Florian Stuker, Christof Baltes, Katerina Dikaiou, Divya Vats, Lucio Carrara, Edoardo Charbon, Member, IEEE, \\ Jorge Ripoll, and Markus Rudin*
}

\begin{abstract}
The high sensitivity of fluorescence imaging enables the detection of molecular processes in living organisms. However, diffuse light propagation in tissue prevents accurate recovery of tomographic information on fluorophore distribution for structures embedded deeper than $0.5 \mathrm{~mm}$. Combining optical with magnetic resonance imaging (MRI) provides an accurate anatomical reference for fluorescence imaging data and thereby enables the correlation of molecular with high quality structural/functional information. We describe an integrated system for small animal imaging incorporating a noncontact fluorescence molecular tomography (FMT) system into an MRI detector. By adopting a free laser beam design geometrical constraints imposed by the use of optical fibers could be avoided allowing for flexible fluorescence excitation schemes. Photon detection based on a single-photon avalanche diode array enabled simultaneous FMT/MRI measurements without interference between modalities. In vitro characterization revealed good spatial accuracy of FMT data and accurate quantification of dye concentrations. Feasibility of FMT/MRI was demonstrated in vivo by simultaneous assessment of protease activity and tumor morphology in murine colon cancer xenografts.
\end{abstract}

Index Terms-Biomedical imaging, magnetic resonance imaging (MRI), optical imaging, optical tomography.

\section{INTRODUCTION}

C OMBINING two or more imaging modalities that provide complementary information on tissue morphology and tissue-specific molecular processes is an attractive concept for improving diagnostic specificity and patient care. For example, the combination of positron emission tomography (PET)

Manuscript received December 14, 2010; accepted January 30, 2011. Date of publication February 10, 2011; date of current version June 02, 2011. This work was supported in part by the Swiss National Foundation under Grant SNF 3100-112835 and Grant 310030-126029, and in part by the European Union under Project FP7 FMT-XCT. Asterisk indicates corresponding author

F. Stuker, C. Baltes, K. Dikaiou, and D. Vats are with the Institute for Biomedical Engineering, University and ETH Zurich, 8093 Zurich, Switzerland (e-mail: stuker@biomed.ee.ethz.ch; baltes@biomed.ee.ethz.ch; dikaiou@biomed.ee.ethz.ch; vats@biomed.ee.ethz.ch)

L. Carrara and E. Charbon are with the Aqua Group, EPFL, 1015 Lausanne, Switzerland (e-mail: ing.luciocarrara@gmail.com; edoardo.charbon@epfl.ch).

J. Ripoll is with the Institute of Electronic Structure and Laser-FORTH, Vassilika Vouton, 71110 Heraklion, Greece (e-mail: jripoll@iesl.forth.gr).

*M. Rudin is with the Institute for Biomedical Engineering, University and ETH Zurich, 8093 Zurich, Switzerland and also with the Institute of Pharmacology and Toxicology, University Zurich, 8057 Zurich, Switzerland (e-mail: rudin@biomed.ee.ethz.ch).

Digital Object Identifier 10.1109/TMI.2011.2112669 and X-ray computed tomography $(\mathrm{CT})$ has emerged as a sensitive tool in cancer diagnostics [1]. More recent developments relate to hybrid PET/MRI systems as clinical and preclinical imaging devices [2]. Combinations with MRI are attractive due to the high quality structural information provided as a result of high soft-tissue contrast. Dedicated MRI methods also yield accurate information on tissue physiology that might be intimately linked to the underlying molecular processes. Moreover, MRI does not involve ionizing radiation like X-ray $\mathrm{CT}$.

Mapping of molecular processes in vivo demands for high sensitivity of the imaging modality as provided e.g., by PET. However, applications of PET may be limited by the short halflife of commonly used positron emitting isotopes, which requires on-site synthesis of PET tracers and correspondingly access to a cyclotron and radiochemistry facility. In contrast, fluorescence markers are in general stable and, when emitting in the near-infrared range, suited for imaging structures deeply embedded in tissue. Therefore, fluorescence imaging has emerged as an alternative to PET for small animal imaging yielding comparable molecular sensitivity. In view of the strengths of the two modalities the combination of MRI and fluorescence imaging constitutes an attractive concept for preclinical research [3].

In designing a hybrid FMT/MRI instrument, two strategies can be pursued: 1) an integrated solution with the FMT setup placed within the magnet or 2) sequential measurements on two independent systems using e.g., an animal support compatible with both modalities to minimize registration errors [4]. Advantages of the integrated approach are that both measurements are inherently registered due to fixed instrument geometry and can be performed simultaneously under identical physiological conditions. Furthermore, the displacement of soft tissues due to movement of the subject between different instruments is avoided. However, several technological challenges are to be met by combining FMT and MRI. In fluorescence imaging, photons emanating from the sample are commonly detected using cooled charge-coupled detectors (CCD), which do not operate at the high magnetic field strengths of animal MRI scanners. Hence, a hybrid system based on CCDs requires the detectors to be located outside of the magnet, and the use of optical fibers for excitation and fluorescence detection [5], [6]. Fiber-based systems are inherently limited by fixed geometry and relatively small number of source-detector pairs [7], which compromises spatial resolution. Moreover, space requirements of fiber bundles may not be compatible with dimensions of high-field small 


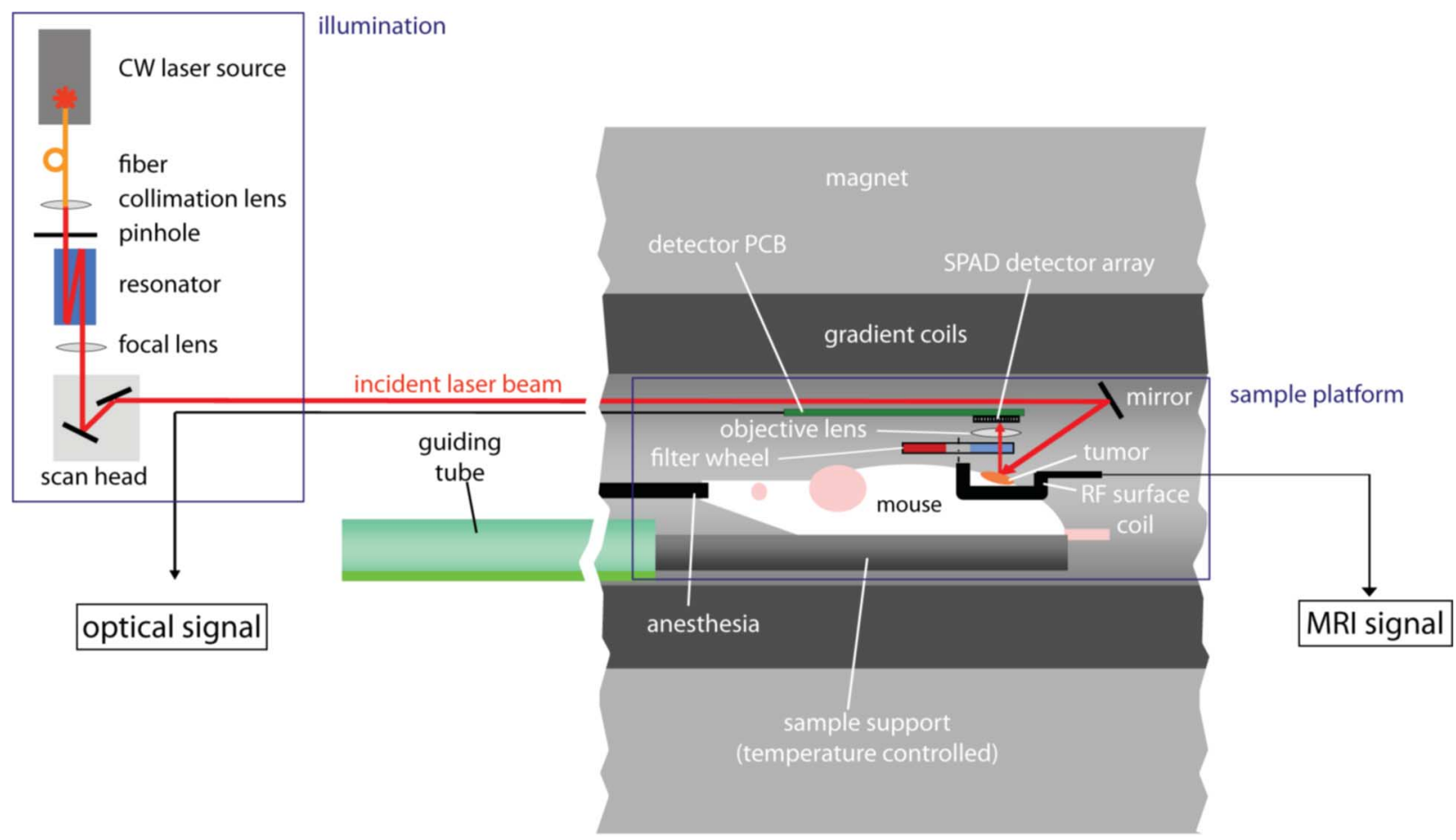

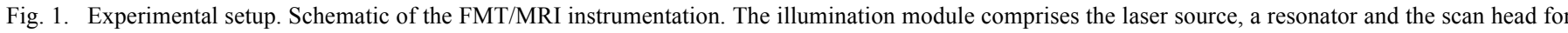

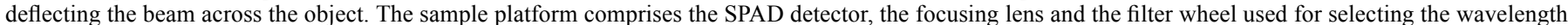
for detection. A semi-cylindrical RF coil is used in transceiver mode. The laser beam is deflected to the sample surface using a mirror.

animal MRI scanners [8]. Finally, signal loss at fiber couplings may be detrimental when guiding low intensity light from the sample surface via an optical fiber to the detector.

Fluorescence detectors that operate in high magnetic fields would enable a fundamentally different solution. Detectors could be positioned in close proximity to the fluorescent source as an integral part of the radio-frequency (RF) antenna used for the detection of MRI signals. Avalanche photodiode detectors are not susceptible to high magnetic fields as demonstrated in PET/MRI systems [9] and are often used in other biomedical applications [10]. In this work we describe a hybrid FMT/MRI system based on a noncontact FMT setup [11], [12] using single-photon avalanche diodes (SPAD) designed to fit inside the $120 \mathrm{~mm}$ gradient bore of a small animal MRI scanner operating at a magnetic field strength of $9.4 \mathrm{~T}$. Devoid of optic fibers, the system offers maximal flexibility with regard to excitation schemes and sample placement. The performance of the hybrid FMT/MRI system was characterized using phantoms with optical parameters mimicking those of biological tissue that comprised one or several fluorescent sources. Aspects investigated were the accuracy of the spatial in-plane and depth information derived from FMT data as well as the sensitivity and linearity of the optical detectors. Feasibility for in vivo imaging was assessed by simultaneously studying protease activity and tumor morphology in a murine colon cancer model.

\section{Materials AND Methods}

\section{A. Instrumentation}

The experimental setup consisted of an illumination module located outside and a sample platform inside the magnet [Fig. 1].
The illumination module consists of a fiber coupled $670 \mathrm{~nm}$ continuous wave laser (B\&W Tek, Newark, DE) for excitation. The multimode fiber (core diameter $100 \mu \mathrm{m}, \mathrm{NA}=0.22$ ) was connected to a numerical aperture matched collimation lens (Thorlabs, Munich, Germany). The pinhole (diameter $0.5 \mathrm{~mm}$ ) behind the lens was mapped with an anti-reflectance coated spherical singlet lens ( $\mathrm{f}=1000 \mathrm{~mm}$, Melles Griot, Bensheim, Germany) by a $2 \mathrm{f}$ image on the subject. To achieve a focal length of $2 \mathrm{~m}$ (required due to the dimensions of the MR magnet) the laser beam was guided through an optical resonator mounted in-between pinhole and focusing lens. The resonator consisted of two coated economy front surface mirrors (Thorlabs, Munich, Germany) placed $400 \mathrm{~mm}$ apart and was traveled five times. The light was then guided to a scan head (Scanlab, Puchheim, Germany), where it was deflected by two galvanometric driven mirrors to allow scanning of sources on the sample surface. These mirrors deflected the beam by $90^{\circ}$ from the input direction. The beam was then directed through an 1800-mm-long tempered epoxy resin tube of $72 \mathrm{~mm}$ inner diameter, which could be separated at the center for better handling. All components were fixed on a movable home-built aluminum breadboard placed outside the bore of the magnet in a magnetic stray field $<10 \mathrm{mT}$ to ensure the proper function of the components.

At distal end of the tube the sample platform was fixed. A coated front surface mirror (Edmund Optics, Karlsruhe, Germany) was placed at the back flange of the sample platform [Fig. 2(a)] located in the isocenter of the MRI, to deflect the beam by an angle of approximately $70^{\circ}$ on the surface of the object, passing through the rectangular window of the MRI transceiver surface coil. A reflection geometry setup was chosen, 


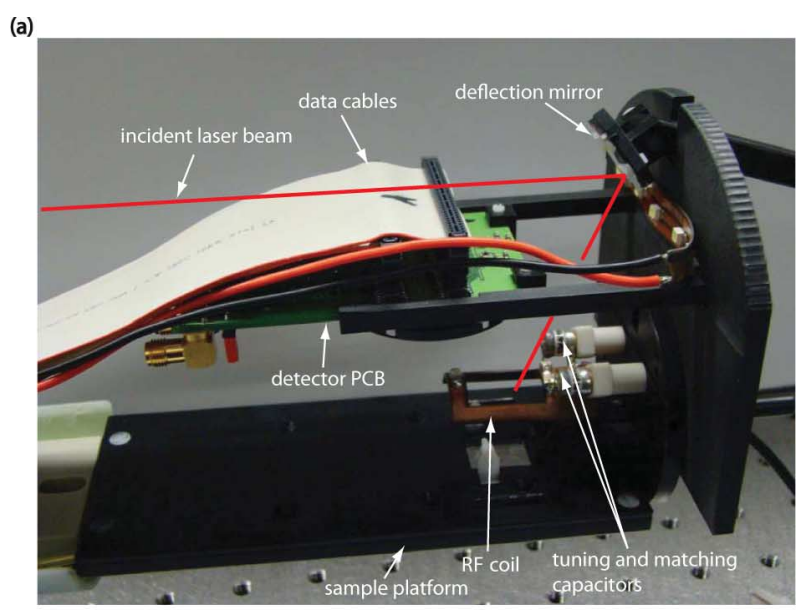

(b)

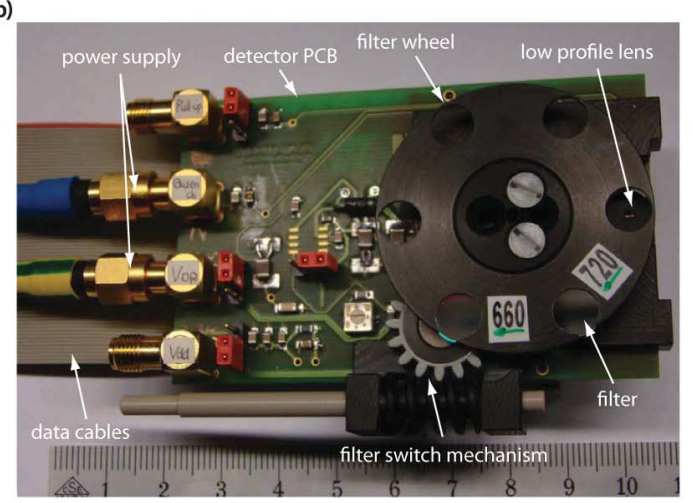

(c)

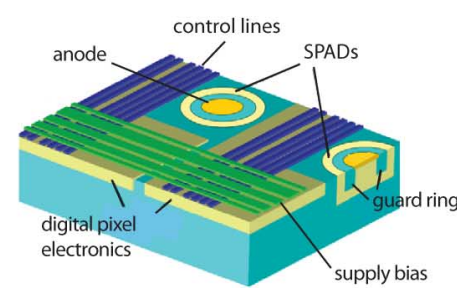

Fig. 2. Setup components. (a) Side view of the sample platform. (b) Bottom view of the detector PCB. (c) Sketch of the SPAD architecture.

which allowed the use of source intensity profiles for image reconstruction [13]. Diffusive light patterns on the surface of the animal were recorded for each illumination point. Light photons emanating from the surface were selected according to their wavelength using filters placed in the filter wheel. High quality bandpass filters (Semrock, Rochester, NY) with peak wavelengths of 660 and $720 \mathrm{~nm}$ for measurements at the excitation and fluorescence wavelength have been used. Filters were characterized by $>90 \%$ transmission over a range of \pm 13 $\mathrm{nm}$. A small 4-mm-diameter fixed focus lens (Edmund Optics, Karlsruhe, Germany) was mounted to the printed circuit board (PCB) in front of the detector array, yielding a field-of-view (FOV) of $8 \times 8 \mathrm{~mm}$ at a focal distance of $33 \mathrm{~mm}$ [Fig. 2(b)]. A custom made $32 \times 32$ array of single-photon avalanche diodes (SPAD) [14] bonded on the PCB was used as photon detector [Fig. 2(c)]. A small LED stripe at the back flange was used for white light illumination during the acquisition of reference surface images. All parts used for the setup were made from materials compatible with operation in high magnetic fields.

\section{B. Detector Details}

The detector consisted of a SPAD array with $32 \times 32$ pixels implemented in a $0.35 \mu \mathrm{m}$ CMOS technology [15]. Each pixel of dimension $30 \times 30 \mu \mathrm{m}^{2}$ comprised a SPAD, a quenching circuit, and a 1-bit counter, all implemented with ten NMOS and two PMOS transistors, resulting in a total pitch of only $30 \mu \mathrm{m}$ and a fill factor of $3.14 \%$ (area ratio). The SPAD is a pn-junction biased above a breakdown voltage $(\mathrm{VBD}=17.7 \mathrm{~V})$ in order to operate in time-uncorrelated photon counting mode. A cathode bias voltage VOP of $21 \mathrm{~V}$ was applied to operate in Geiger mode yielding an excess bias voltage VE of $3.3 \mathrm{~V}$. At this excess value, a maximum photon detection probability of $35 \%$ (fill factor not included) was measured with a sensitivity spectrum ranging from 350 to $850 \mathrm{~nm}$. Calculating the overall sensitivity (fill factor times photon detection probability) revealed that about one in one hundred photons is detected. The image sensor array operates according to the rolling shutter principle. Each row is selected via the row decoder and read independently for all the columns during one clock cycle. The row is subsequently reset for initiating a new integration period. A clock frequency of $48 \mathrm{MHz}$ was used due to firmware limitations, thus the minimum integration time in our experiment was $2.66 \mu \mathrm{s}$. The total exposure time was set by the selected iterations to build a frame. Since each pixel produces single bit digital information, an external FPGA chip-set is used to reconstruct a multi-bit image by incrementing 1024 32-bit counters sequentially row-by-row. The chip-set was not designed to tolerate high magnetic fields and was thus placed outside the bore of the magnet along with the circuit needed for communication with the computer. The connection between the chip-set and PCB was achieved via conventional flat ribbon cables. The SPAD detector array was tested inside the MRI system to assess the potential interference of a high static magnetic field of $9.4 \mathrm{~T}$. The signal-to-noise ratio (SNR) of $45 \mathrm{~dB}$ is dominated by Poisson noise, while the maximum dynamic range achieved at 12 frames per second was $90 \mathrm{~dB}$. The median of the noise dark count rate (DCR) per pixel measured over the entire array is $140 \mathrm{~Hz}$ at room temperature and can be reduced by cooling of the chip to $-40^{\circ} \mathrm{C}$ to DCR a median of $98 \mathrm{~Hz}$. DCR was unaffected by the magnetic field.

\section{MRI Details}

All MR experiments were carried out on a Bruker BioSpec 94/30 (Bruker BioSpin MRI, Ettlingen, Germany) horizontal small animal MR system operating at $400 \mathrm{MHz}$ equipped with a gradient system capable of generating a maximum strength of $400 \mathrm{mT} / \mathrm{m}$ with a minimum rise time of $80 \mu \mathrm{s}$. A home-built rectangular surface coil $(20 \times 24 \mathrm{~mm})$ was used for RF signal transmission and reception. The coil made of a flexible PCB substrate with standard copper layer was designed for mouse imaging and therefore curved to a cylindrical radius of $15 \mathrm{~mm}$. A window of $13 \times 20 \mathrm{~mm}$ was cut out of this single loop coil to allow for optical measurement (illumination and detection) in 
reflectance mode from the top. Three-dimensional MR imaging was performed using a gradient echo sequence with the parameters: field of view FOV $=40 \times 40 \times 16 \mathrm{~mm}^{3}$; matrix dimensions MTX $=80 \times 80 \times 32$; voxel dimension VOX $=$ $500 \times 500 \times 500 \mu \mathrm{m}^{3}$; excitation pulse angle $\alpha=25^{\circ}$; echo time/repetition time TE/TR $=3.9 / 90 \mathrm{~ms}$; number of averages $\mathrm{NA}=4$ resulting in a scan time $=15 \min 22 \mathrm{~s}$. In addition separate acquisitions with zero excitation pulse angle were performed for estimation of noise levels. In vivo experiments were performed using a two-dimensional multislice MR sequence with the following parameters: 14 slices of $700 \mu \mathrm{m}$ thickness; FOV $=30 \times 20 \mathrm{~mm}^{2} ;$ MTX $=200 \times 133 ;$ pixel dimension PIX $=150 \times 150 \mu \mathrm{m}^{2} ;$ TE $/ \mathrm{TR}=5.0 / 250 \mathrm{~ms} ; \mathrm{NA}=20$, yielding a total acquisition time of $11 \mathrm{~min} 5 \mathrm{~s}$.

\section{Phantom Preparation}

For the phantom experiments two tissue-mimicking siliconebased phantoms were prepared. Phantom (A) was used to characterize spatial resolution and concentration accuracy of the optical system and for the combined measurements with the MRI. It comprised holes for dye administration separated with center-to-center distances of $2,3,4$, and $5 \mathrm{~mm}$ in a plane parallel to the surface at a depth of $1 \mathrm{~mm}$. Phantom (B) was used for depth analysis and comprised holes parallel to the surface at depths of 1,2, and $3 \mathrm{~mm}$ with respect to the surface with an in-plane separation of $5 \mathrm{~mm}$. The holes could be filled with fluorescent dyes. Both phantoms were based on a room temperature vulcanizing (RTV) silicone (Wacker Silicone, Munich, Germany). To simulate optical tissue properties $\left(\mu_{a}=0.2 \mathrm{~cm}^{-1}\right.$ and $\mu_{s}^{\prime}=10 \mathrm{~cm}^{-1}$ ), $\mathrm{TiO}_{2}$ particles (Alfa Aesar, Karlsruhe, Germany) and carbon black powder (Alfa Aesar, Karlsruhe, Germany) were added as scattering and absorption agents, respectively. Resulting optical properties were measured using a near infrared spectrometer (OxiplexTS, Champaign, IL) at $690 \mathrm{~nm}$. The phantoms were cuboid shaped with a thickness of $10 \mathrm{~mm}$ and contained holes of $1.5 \mathrm{~mm}$ diameter for administration of the fluorescent dye at chosen positions. The dye was filled into small glass capillaries (outer diameter: $1.5 \mathrm{~mm}$ inner diameter: $0.6 \mathrm{~mm}$ ) and inserted into the holes.

\section{E. Subcutaneous Tumor Animal Model}

Pathogen-free female BALB/C (CAnN.Cg-Foxn1 $\langle\mathrm{nu}\rangle / \mathrm{crl})$ nude mice (8-10 week old, weighing $20-25 \mathrm{mg}$ ) were obtained from Charles River Laboratory (Sulzfeld, Germany) and housed in a controlled environment $\left(23^{\circ} \mathrm{C}, 12 \mathrm{~h} / 12 \mathrm{~h}\right.$ light/dark cycle) with unlimited access to water and chlorophyll free food (Kliba Nafag, Kaiseraugst, Switzerland). All experiments were performed in accordance to the Swiss Veterinary law (License no. ZH 172-2008. C51-cells, a colon cancer-derived secondary cell-line, were used to generate tumors. $1 \cdot 10^{6}$ cells were injected subcutaneously on the left thigh flank of the nude mice. The animals were monitored every second day for their body weight and tumor size. Animals with tumors of a size $3 \times 3 \mathrm{~mm}^{2}$ and larger were selected for imaging experiments.

\section{F. Fluorescent Agents}

The commercially available cathepsin-activatable near in frared (NIR) fluorescent imaging agent ProSense 680 (VisEn
Medical, Bedford, MA), a fluorophore conjugated graft polymer of polyethylene glycol (PEG) and poly-lysine, was administered intravenously via the tail vein in all in vivo imaging studies. A single dose of $13 \mathrm{nmol}$ was given followed by a second dose of $13 \mathrm{nmol}, 24 \mathrm{~h}$ after the first injection. The FMT/MRI measurements were done $24 \mathrm{~h}$ after the second dose of ProSense 680.

The near-infrared fluorescent oxazine dye AOI987 used in the phantom experiments was prepared according to published procedures [16].

\section{G. Fluorescence Data Acquisition}

The silicone-based phantoms were used for characterizing the performance of the FMT/MRI system. For each measurement, one or two fluorescent samples consisting of an aqueous solution of AOI987 [16] at various concentrations were included at defined depths and with variable spacing. Initially the sample was placed on the imaging platform within the FOV of the optical system and under the RF surface coil of the MRI system. For excitation, the laser beam was scanned across a grid with $7 \times 7$ illumination spots with dimensions and the exact location of the grid adjusted operator-interactively in order to match the ROI on the sample surface. We have chosen an integration time of 2-4 s per source position depending on the fluorescent signal strength. This results in a total acquisition time between 5 and $9 \mathrm{~min}$. For each source position an image was recorded resulting in a $\mathrm{N}_{\mathrm{x}} \times \mathrm{N}_{\mathrm{y}} \times \mathrm{N}_{\mathrm{s}}$ array of images, where $\mathrm{N}_{\mathrm{x}}$ and $\mathrm{N}_{\mathrm{y}}$ are the number of pixels in $\mathrm{x}$ and $\mathrm{y}$ and $\mathrm{N}_{\mathrm{s}}$ the number of sources. For calibration purposes the sources were measured on a white paper placed in the focal plane of the camera in order to get the center position of the sources and the pixel dimensions in centimeters. After that the optical acquisition was started and images were recorded at two wavelengths corresponding to the excitation $(660 \mathrm{~nm})$ and emission maximum $(720 \mathrm{~nm})$ of AOI987.

For in vivo measurements the illumination scheme and filter settings were kept the same.

\section{H. Fluorescence Data Reconstruction}

For image reconstruction the normalized Born approximation was used, which has been shown to yield robust data by considering the ratio of the measured emission and excitation images [17]. The values of this ratio were used in the forward model [18] accounting for the fluorescence signal on the sample surface. The reconstruction was performed by inverting this forward model using an algebraic reconstruction technique (ART). This algorithm is an iterative method used in computed tomography [19] and adapted for imaging diffusive media with boundary restrictions [20]. An initial assumption for the fluorophore distribution is iteratively improved using a least square minimization procedure to minimize the difference between reconstructed and measured data. The reconstruction yielded a three dimensional map of the fluorescent source distribution within the subject. Simulations were performed using the same forward model and reconstruction algorithm.

\section{RESULTS}

Quantitative analyses have been carried out for assessing the crosstalk between modalities and the accuracy of in-plane and 
(a)

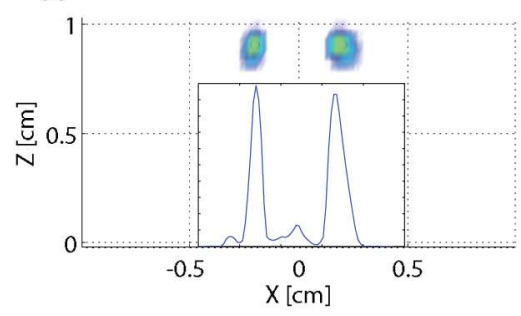

(d)

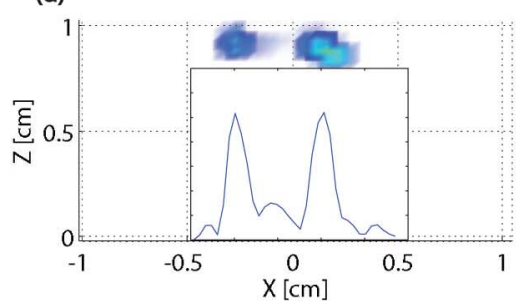

(b)
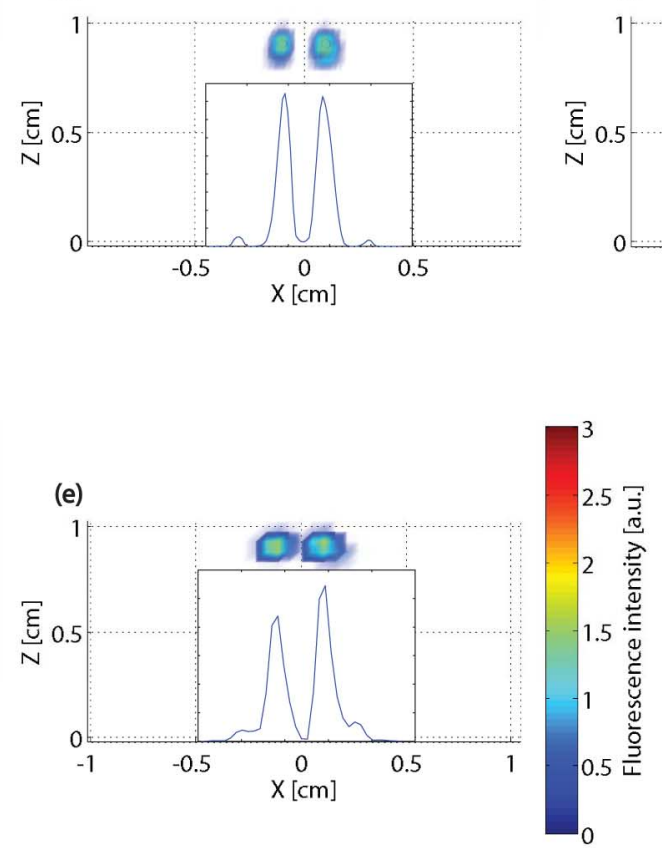

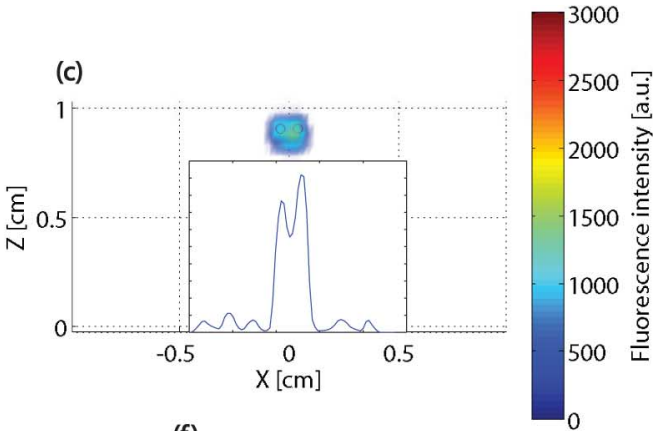

(f)

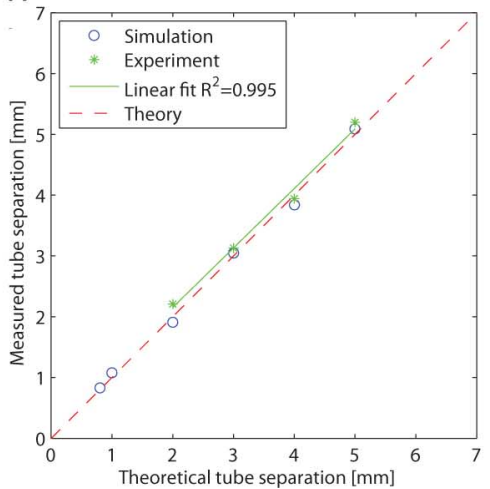

Fig. 3. Spatial resolution in a phantom. (a)-(c) Reconstructed fluorescence intensity (arbitrary units) from simulated data in xz-projection for $4 \mathrm{~mm}$ (a), $2 \mathrm{~mm}$ (b) and $0.8 \mathrm{~mm}$ (c) center-to-center tube separation. The tubes are indicated as red circles. The insets show the line profiles at $\mathrm{z}=9 \mathrm{~mm}$. (d), (e) Reconstructed fluorescence intensity (arbitrary units) from experimental data in xz-projection for $4 \mathrm{~mm}$ (d) and $2 \mathrm{~mm}$ (e) center-to-center distance. The inset show the line profiles at $z=9 \mathrm{~mm}$. (f) Plot of the extracted tube separations for simulated data (blue circles) and experimental data (green stars) versus the theoretical separations (red dashed line).

depth reconstruction. Moreover the linearity between the fluorescence intensity and the local dye concentration was investigated. Finally simultaneous hybrid measurements were performed with the combined system in a phantom experiment and in a first in vivo animal study.

\section{A. Crosstalk}

In hybrid systems potential crosstalk between the imaging modalities is an issue, i.e., sensitivity might be compromised as compared to the sensitivity of standalone devices. Also, interference might cause image artifacts or image distortions. We calculated the signal-to-noise ratio (SNR) of the FMT by dividing the signal of a ROI in fluorescent raw image by the standard deviation of noise value extracted from a ROI in a background image, which was recorded in the absence of fluorescence excitation, and where only ambient light and detector noise contribute to the signal. The SNR was evaluated using the previously described phantom prior $\left(\mathrm{SNR}_{\mathrm{opt}}=3.58 \pm 0.52\right)$ and during the MR image acquisition $\left(\mathrm{SNR}_{\mathrm{opt}}=3.67 \pm 0.39\right)$ and we did not observe any degradation in $\mathrm{SNR}_{\mathrm{opt}}$ within error limits due to insertion of the optical setup into the MRI system. Similarly, the SNR of the MR measurements was evaluated with and without simultaneous optical measurement $\left(\mathrm{SNR}_{\mathrm{MRI}}=64.41 \pm 2.51\right.$ versus $\mathrm{SNR}_{\mathrm{MRI}}=63.20 \pm 2.35$ ), which did not reveal an effect on the MRI sensitivity. For MRI SNR calculations the mean signal intensity in a sample ROI located at a fixed location within the FOV was divided by the standard deviation of the intensity in a ROI comprising only contributions from noise. Moreover, we did not observed any artifacts introduced by the optical detector in the MR images since the SPAD was positioned at a distance of $40 \mathrm{~mm}$ from the RF coil and outside the FOV of the MRI data acquisition. Correspondingly, potential artifacts caused by the SPAD were not visible in the MR images. All MR sequences can be run by the setup, the only limitation is the inhomogeneous excitation by the surface coil. We therefore conclude that crosstalk between modalities in FMT/MRI system is negligible.

\section{B. Spatial Resolution}

A critical parameter in imaging is spatial resolution. In MRI voxel dimensions are typically of the order of 150-500 $\mu \mathrm{m}$ depending on the acquisition parameters, whereas resolution in FMT is inferior, typically $1-2 \mathrm{~mm}$ isotropic. The FMT spatial resolution in our hybrid system was evaluated using phantom (A) comprising two identical fluorescent tubes with variable spacing $(2-5 \mathrm{~mm})$. Simulations revealed that sources separated by more than $0.8 \mathrm{~mm}$ could be clearly discriminated [Fig. 3(a)-(c)] by analyzing the line profile in $\mathrm{x}$ direction. To analyze the line profile the reconstructed volume was projected to the xz-plane. The line profile is drawn through the peak value of the embedded tubes. To determine the spatial separation a gauss fit was applied to each peak in the line profiles and the distance between the peak value of the two fitted Gaussian profiles was taken as the separation distance. If two clearly distinguishable peaks could be identified in the line profile the two signals were considered as spatially separable. Experiments were limited by the diameter and the wall thickness of the capillaries used. The experimental dispersion of the fluorescence 
intensity was slightly larger than predicted by the simulations [Fig. 3(d)-(e)] and some artifacts were observed, which are a result of imperfect reconstruction due to deficiencies like the forward model (assuming a fluorescence point source embedded in an optically homogeneous medium), data collection (detector noise) and SNR which contributes to the uncertainty of the data lead therefore to these artifacts. Comparing the full widths at half maximum of the FMT/MRI setup for simulated and experimental data, we estimate the FMT in-plane spatial resolution of the FMT/MRI setup to be $0.9 \mathrm{~mm}$. A linear correlation has been found between reconstructed experimental data and true tube separation $\left[\mathrm{R}^{2}=0.995\right.$, slope $=0.978$; Fig. 3(f) $]$ indicative of the accuracy of the in-plane spatial localization.

\section{Depth Resolution}

Quantitative analysis of dye concentrations depends on accurate reconstruction of the depth of the fluorescent source, which was analyzed for a depth range of 1-3 $\mathrm{mm}$ using both simulations and experimental data. To analyze accuracy of depth information a line profile was drawn in $z$ direction across the maximum intensity of the volume projected onto the xz-plane. The distance between the phantom surface and the peak value of a fitted Gaussian line was taken as a measure of depth. For this experiment phantom (B) was used comprising one fluorescent tube in the investigated depth. The dispersion of fluorescence intensity increases slightly with increasing depth [Fig. 4(a)-(d)], as photon scattering scales with their path length through tissue. The observed artifact in Fig. 4(c) is a result of imperfect reconstruction due to different deficiencies like the forward model, data collection and SNR which contributes to the uncertainty of the data as already discussed. Comparing reconstructed with true depths demonstrated a good correlation up to depth values of about $3 \mathrm{~mm}$ [Fig. 4(e)]. If depth of the fluorescent source exceeds $3 \mathrm{~mm}$, the actual depth is underestimated in the reconstructed image data sets. This can be attributed to reflection geometry setup, which emphasizes contributions from superficial structures. Simulated data reveal that the limited field of view in the current setup has only minor influences on the accuracy of depth localization. By keeping the imaging parameters constant and doubling the FOV the accuracy of the depth localization of the FMT setup only slightly increases by $2 \%$.

\section{Quantification Analysis}

A principal motivation for using tomographic imaging is the ability to derive quantitative information on the local dye concentrations. Two aspects are of interest: 1 ) the minimal dye concentration that can be detected (detection limit), and 2) the linearity of the relationship fluorescence intensity versus dye concentration. Experiments were carried out using a tissue phantom with a fluorescent source embedded at a depth of $1 \mathrm{~mm}$. The correlation between AOI987 concentration and fluorescence intensity reconstructed revealed a linear relationship $\left[\mathrm{R}^{2}=0.978\right.$; Fig. 4(f)] for a range from 0.36 to 25 picomoles, with a detection limit of approximately 0.3 picomoles of AOI987 dissolved in a volume of $1.5 \mu 1$.
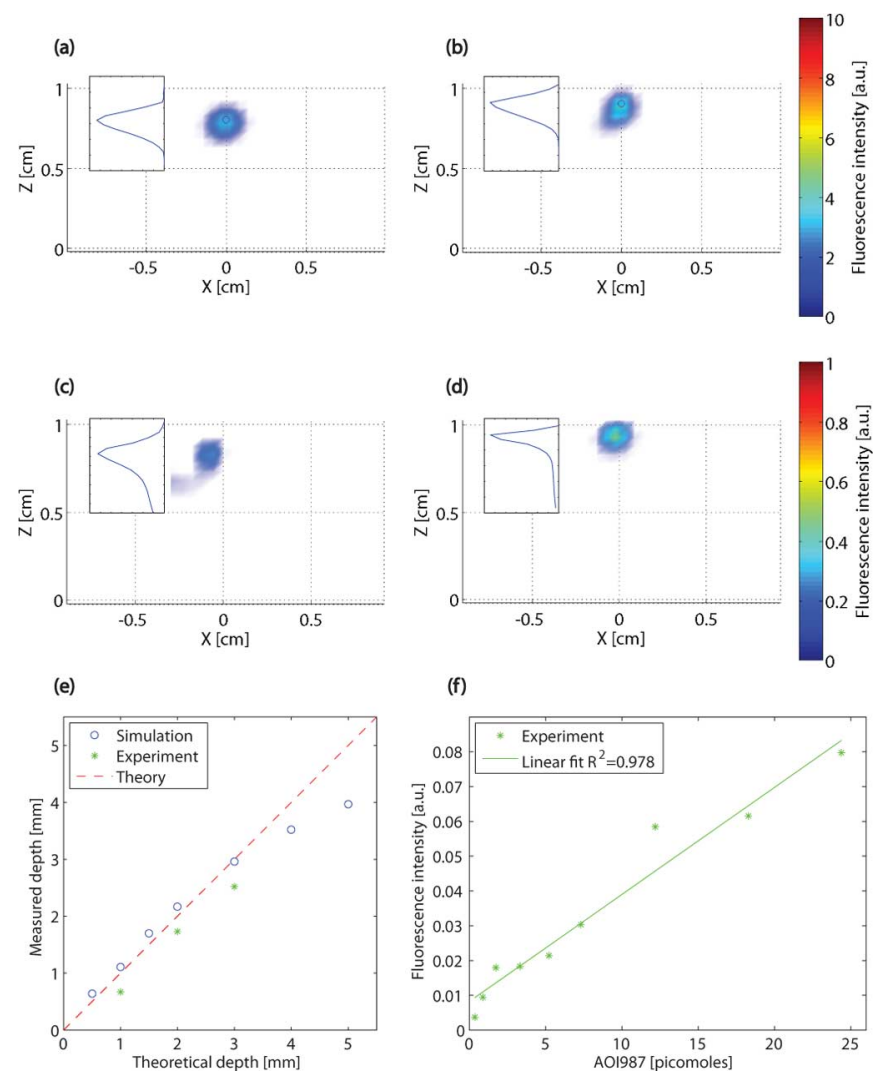

Fig. 4. Depth resolution in a phantom. (a), (b) Reconstructed fluorescence intensity (arbitrary units) from simulated data in xz-projection for $2 \mathrm{~mm}$ (a) and $1 \mathrm{~mm}$ (b) below the surface. The insets show the depth line profiles. The tubes are indicated as red circles. (c), (d) Corresponding reconstructed fluorescence intensity (arbitrary units) from experimental data in xz-projection for $2 \mathrm{~mm}$ (c) and $1 \mathrm{~mm}$ (d) depth. The inset show the depth line profiles. (e) Plot of the extracted tube depth for simulated data (blue circles) and experimental data (green stars) versus theoretical depth (red dashed line). (f) Reconstructed mean fluorescence intensity versus AOI987 concentration in the tube of the phantom

\section{E. Hybrid Imaging}

The feasibility of hybrid FMT/MRI was investigated using a tissue phantom [Fig. 5(a)-(d)] and in vivo using tumor bearing mice [Fig. 5(e)-(j)]. Due to the fixed geometry of the two detector systems a phantom measurement using fluorescent tubes visible in both modalities enabled the definition of the affine transformation to register the FMT data using the MRI images as reference coordinate system. Voxel dimensions and the slice positions for the FMT data were adjusted accordingly. The same affine transformation could then be applied to in vivo data sets as the FMT/MRI geometrical arrangement was left unchanged.

For these experiments the acquisition time for both modalities for phantom and in vivo measurements were on the same time scale allowing to measure time dependent processes simultaneously.

A sketch of the silicone slab used for phantom experiments is shown in Fig. 5(a) where one of four holes is filled with the fluorescent dye and the slice position is indicated. It was shown that the overlay image of MRI [Fig. 5(b)] and FMT [Fig. 5(c)] sections revealed an accurate localization of the reconstructed fluorescent tube from the FMT data [Fig. 5(d)]. For in vivo proof-of-principle of simultaneous FMT/MRI measurements a murine tumor model was used. C51 cells (colon cancer-derived cell-line) were 
(a)

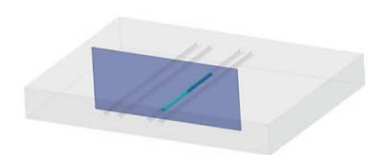

(b)

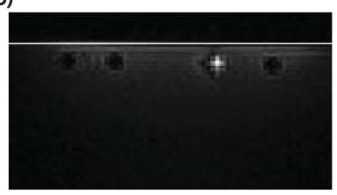

(c)

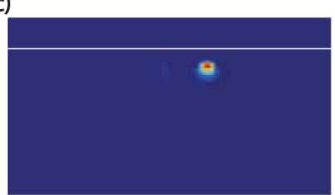

(d)

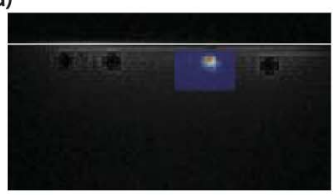

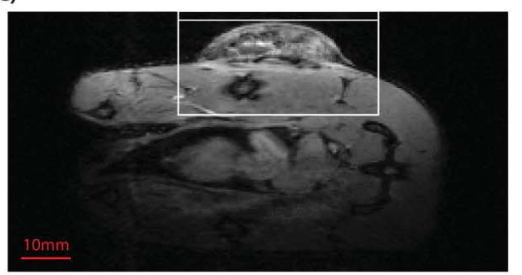

(f)

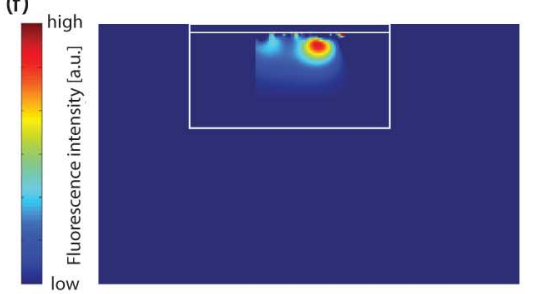

(g)

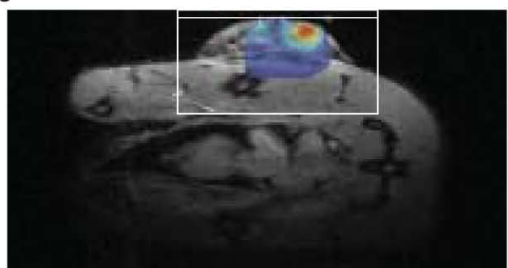

(h)

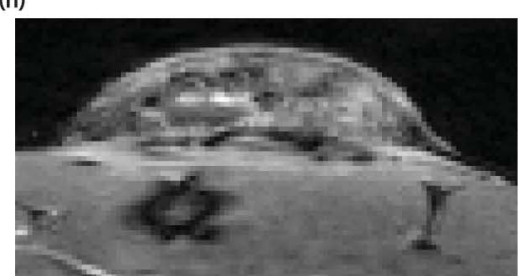

(i)

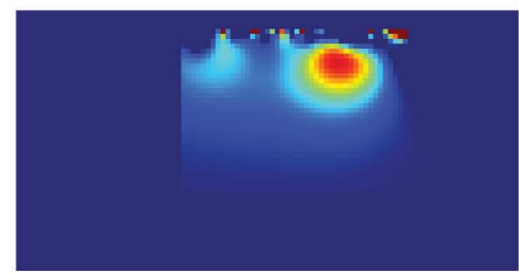

(j)

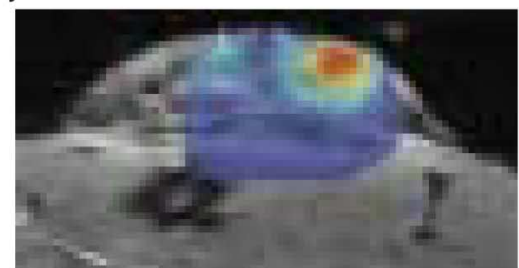

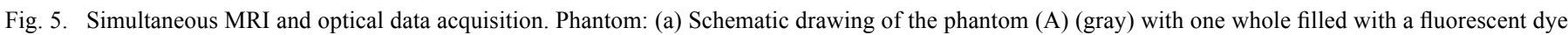

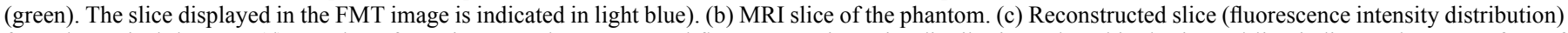

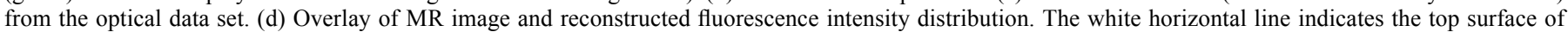

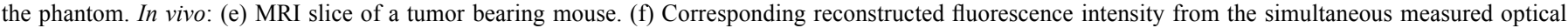

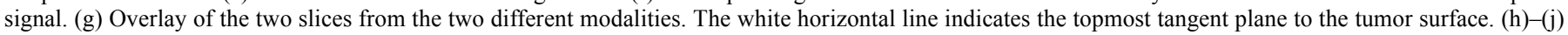
Detailed view of the tumor region.

subcutaneously implanted in the flank of a nude BALB/C mouse. The majority of tumors are known to express high levels of proteases (protein degrading enzymes), which are involved e.g., in the degradation of the extracellular matrix to enable tissue invasion and metastasis formation [21]. An activatable probe (ProSense 680) that generates fluorescent signals only upon cleavage by proteases [22], [23] was used to assess the level of protease activity by FMT. A representative MR image [Fig. 5(e)] and the corresponding FMT section displaying fluorescence intensity reflective of protease activity [Fig. 5(f)] have been registered [Fig. 5(g)]. They show regions of high protease activity within the tumor tissue. The edge of the slab used for the FMT reconstruction corresponded to the tumor tangential plane in the MR image oriented parallel to the focal plane of the detector [indicated by the white horizontal line in Fig. 5(e)-(g)]. An enlarged region of the registered FMT and MR image gives an insight into the fluorescence dye distribution (protease activity) within the structural image provided by MR [Fig. 5(h)-(j)]. Interestingly, protease activity measured in FMT was not homogeneous across the tumor. Histological analysis confirmed heterogeneity of protease activity throughout the tumor.

\section{DISCUSSION}

Proper interpretation of molecular and/or cellular information derived from molecular imaging studies requires the registration to an anatomical reference that is commonly recorded using different imaging modalities. A straightforward solution is a sequential imaging strategy, which requires a sample support that is compatible with both modalities and that comprises fiducial markers as landmarks for the registration process. An advantage of this setup is that it provides optimal performance of each standalone system without compromising image quality. Disadvantages are that sequential measurements take inherently longer which puts demands regarding the maintenance of physiologically stable conditions throughout the measurement period. In addition, when studying fast concurrent processes with different modalities, sequential sampling is not feasible. Moreover, soft-tissue may move during the translocation between imaging modalities, which demands for nonrigid body registration procedures that are intrinsically difficult. Thus true hybrid systems are highly attractive for biomedical research applications, as they provide simultaneous multiplexed information in an inherently registered manner due to concurrent measurement with both modalities in a rigid configuration. Combinations with MRI, which provides a high soft-tissue contrast and has become a standard method both in preclinical and clinical structural and functional imaging, are challenging due to the hostile environment of a high magnetic field. Hybrid optical imaging-MRI systems are typically fiber-based and used in combination with large bore clinical MRI with gradient systems of 50-60 cm inner diameter to account for the space requirements by the source and detector fiber bundles. The use of a clinical scanner for studies in small rodents is suboptimal: lower field strength and weaker magnetic field gradients would compromise the spatial resolution achievable. This might be sufficient if MRI data are only used as reference indicating major anatomical structures; yet studies with MRI providing complementary information as independent imaging modality will suffer. Moreover, the rigid fiber geometry limits the flexibility of the optical imaging 
setup: the number of source-detector pairs is limited, definitely much smaller than for typical array detectors (with typically $>1$ $\mathrm{k}$ pixels) and the excitation schemes/points are predefined by the position of excitation fibers. Fiber-based systems are typically operated with the light guiding fibers in direct contact with the sample. This renders the experimental procedure cumbersome requiring manual adjustments of individual fibers. The non-contact system presented in this work allows overcoming these limitations. Excitation can be varied by adjusting the parameters of the scanning device, while the fixed distance between the SPAD array detector and the MRI surface coil, which is directly placed over the animal's body surface, ensures maintenance of optical focus (apart from the surface curvature) irrespective of the body region sampled. The focal plane was set according to a tangential plane on the animal surface perpendicular to the objective lens.

In a hybrid system combining two complementary imaging modalities, system performance of each modality should be not or only minimally degraded with regard to their standalone performance, though some performance loss as compared to optimized standalone systems, which might use different detector technologies, might be acceptable in view of the benefits of the multimodality readouts. In a combined FMT/MRI system, the MRI setup defines the design of the integrated FMT system: the detector must be functional at high magnetic fields and has to fit into the small bore of the gradient system $(120 \mathrm{~mm}$ inner diameter in our case). The use of an MRI-compatible SPAD array comprising $32 \times 32$ detectors led to some loss in performance as compared to standalone FMTs using cooled CCD cameras with regard to sensitivity, field-of-view and the number of source-detector pairs available. Nevertheless, the setup is still superior to fiber-based rigid geometry systems with regard to experimental flexibility and amount of independent data generated for reconstruction. Moreover, its performance is expected to improve with new generation SPAD arrays. The spatial resolution provided by the FMT/MRI setup was found to be comparable to that of microPET systems [24], [25]: the in-plane spatial resolution derived from phantom experiments was at least $2 \mathrm{~mm}$, whereas simulations indicated that it should be possible to resolve two fluorescent sources of equal strength separated by $0.8 \mathrm{~mm}$. The linear dependence of measured and theoretical source separation for a range of $0.8-5 \mathrm{~mm}$ center-to-center distance reflects the accuracy of the reconstruction algorithm. On the other hand, the estimation of the source depth from the sample surface was found to be systematically smaller than the theoretical depth both for simulated and experimentally found data for depth values exceeding $3 \mathrm{~mm}$. Two factors contribute to this: Due to diffusive light propagation the intensity is reduced to a level that makes detection difficult (in particular in view of autofluorescence contributions from surface structures) and secondly, the small field-of-view prevents accurate sampling of the intensity distribution of the surface which is critical for proper reconstruction of the source location and distribution. Though simulations have revealed that by increasing the field of view to $16 \times 16 \mathrm{~mm}$, the accuracy of depth reconstruction can only be marginally improved.

The claim of molecular imaging is to provide noninvasive quantitative information on molecular processes in the intact organism. These events commonly occur at a low frequency; hence any molecular imaging modality should provide inherent high sensitivity. Calibration experiments revealed that the FMT/MRI system was capable of detecting subpicomole amounts of dye. Moreover, fluorescence intensity depended linearly on the amount of fluorescent dye in the sample volume, an important prerequisite for quantitative studies. The amount of dye used covered the ranges typically reported for in vivo applications.

The true strength of a hybrid system is that high resolution structural images can be annotated with molecular information derived from an inherently registered data set. The in vivo study, which allowed identifying regions within a subcutaneous tumor that are high in protease activity, clearly illustrates the potential of the approach. While the MRI signature clearly indicates tumor heterogeneity based on differences in MRI contrast (dominated by differences in $T_{1}$ relaxation times), the FMT information adds a molecular component to it: high protease activity is commonly associated with invasive (malignant) tumor behavior [26]. The heterogeneity of fluorescence activity confirmed by histological analysis reflects the sensitivity of our hybrid imaging system. Even more attractive FMT/MRI applications would be the correlations between physiological/functional MRI readouts (e.g., vascular permeability as a target for tumor angiogenesis [27]) with FMT derived molecular information which demand for simultaneous measurement. The prerequisite for such applications is that the time resolution of the slower of the two modalities is still sufficient to capture the biological process. Sequential measurements involving transfer of the sample from modality A to B might not be possible, as this would be too time consuming (including adjustments of individual modalities) so the sample state would be different.

Aside from collecting multiplexed data, hybrid systems can offer improved data reconstruction for the low-resolution modality, in our case for FMT. This has been used in PET/CT systems, where the CT map is used to compute the tissue attenuation correction required for reconstructing PET data [28]. Correspondingly, in an FMT/MRI system, the anatomical information provided by MR can be fed into both the forward and the backward model of the FMT reconstruction [29], which should render the FMT reconstruction more accurate. However, the use of imaging priors bears the risk of over-constraining the FMT reconstruction.

\section{CONCLUSION}

In conclusion, a novel hybrid setup for simultaneous acquisition of FMT and MRI data compatible for a small animal MRI system has been described (Figs. 1 and 2). The interference between the two modalities was found negligible. Reconstruction of fluorescence data revealed accurate localization of fluorescent sources, whereas the spatial resolution was demonstrated experimentally to be in the order of $2 \mathrm{~mm}$ (see Fig. 3) whereas based on the width of the individual profile we estimated that sources separated by $0.9 \mathrm{~mm}$ should be distinguishable. Limitations in depth resolution may be attributed to the inherent surface weighting of the reflectance geometry approach and to the limited array size $(32 \times 32$ pixels $)$ and the limited field of view of the current detector array. Improvements in depth reconstruction might therefore be achieved by using the setup in 
transmission mode, which would in addition reduce the interference by autofluorescence. The next generation of detector arrays will also have significantly increased matrix dimensions and should due to the increased number of source detector pair improve reconstruction accuracy. Hybrid FMT/MRI is sensitive and we determined a sub-picomole detection limit for sources embedded at a depth of $1 \mathrm{~mm}$ below the surface [Fig. 4(f)]. For this imaging domain the system performance was found to be quantitative, as reflected by the linear relationship of fluorescence signal versus dye concentration. With its potential for annotating structural/physiological with quantitative molecular information FMT/MRI constitutes an attractive hybrid modality for experimental biomedical researchers.

\section{ACKNOWLEDGMENT}

The authors gratefully acknowledge the contribution of R. Keist for cell culturing, K. H. Altmann for AOI987 preparation and $\mathrm{C}$. Ruettimann for fruitful discussions.

\section{REFERENCES}

[1] D. W. Townsend, J. P. J. Carney, J. T. Yap, and N. Hall, "PET/CT today and tomorrow," J. Nucl. Med., vol. 45, no. 1, pp. 4S-14S, Jan. 2004.

[2] M. S. Judenhofer, C. C. Catana, B. K. Swann, S. B. Siegel, W.-I. Jung, R. E. Nutt, S. R. Cherry, C. D. Claussen, and B. J. Pichler, "PET/MR images acquired with a compact MR-compatible PET detector in a 7-T magnet," Radiology, vol. 224, no. 3, pp. 807-814, Sep. 2007.

[3] M. Niedre and V. Ntziachristos, "Elucidating structure and function in vivo with hybrid fluorescence and magnetic resonance imaging," in Proc. IEEE, Mar. 2008, vol. 96, no. 3, pp. 382-396.

[4] M. Allard, D. Côté, L. Davidson, J. Dazai, and R. M. Henkelman, "Combined magnetic resonance and bioluminescence imaging of live mice,” J. Biomed. Opt., vol. 12, no. 3, p. 034018, May/Jun. 2007.

[5] H. Xu, R. Springet, H. Dehghani, B. W. Pogue, K. D. Paulsen, and J. F. Dunn, "Magentic-resonance-imaging-coupled broadband near-infrared tomography system for small animal brain studies," Appl. Opt., vol. 44, no. 11, pp. 2177-2188, Apr. 2005.

[6] G. Gulsen, O. Birgul, M. B. Unlu, R. Shafiiha, and O. Nalcioglu, "Combined diffuse optical tomography (DOT) and MRI system for cancer imaging in small animals," Technol. Cancer Res. Treatment, vol. 5, no. 4, pp. 351-363, Aug. 2006.

[7] A. M. Siegel, J. B. Mandeville, and D. A. Boas, "Temporal comparison of functional brain imaging with optical tomography and fMRI during rat forepaw stimulation," Phys. Med. Biol., vol. 48, no. 10, pp. 1391-1403, 2003.

[8] S. C. Davis, B. W. Pogue, R. Springett, C. Leussler, P. Mazurkewitz, S. B. Tuttle, S. L. Gibbs-Strauss, S. S. Jiang, H. Dehghani, and K. D. Paulsen, "Magnetic resonance-coupled tomography scanner for molecular imaging of tissue," Rev. Sci. Instrum., vol. 79, no. 6, p. 064302 , 2008.

[9] B. J. Pichler, M. S. Judenhofer, C. Catana, J. H. Walton, M. Kneilling, R. E. Nutt, S. B. Siegel, C. D. Claussen, and S. R. Cherry, "Performance test of an LSO-APD detector in a 7-T MRI scanner for simultaneous PET/MRI," J. Nucl. Med., vol. 47, pp. 639-647, 2006.

[10] E. Grigoriev, A. Akindinov, M. Breitenmoser, S. Buono, E. Charbon, C. Niclass, I. Desforges, and R. Rocca, "Silicon photomultipliers and their bio-medical applications," Nucl. Instrum. Methods Phys. Res. A, vol. 571, pp. 130-133, 2007.

[11] J. Ripoll, R. B. Schulz, and V. Ntziachristos, "Free-space propagation of diffuse light: Theory and experiments," Phys. Rev. Lett., vol. 91, no. 10, p. 103901, Sep. 2003.
[12] A. Martin, J. Aguirre, A. Sarasa-Renedo, D. Tsoukatou, A. Garofalakis, H. Meyer, J. Ripoll, and A. M. Planas, "Imaging changes in lymphoid organs in vivo after brain ischemia with three-dimensional fluorescence molecular tomography in transgenic mice expressing green fluorescent protein in t lymphocytes," Molecular Imag., vol. 7, no. 4, pp. 1-11, Jul./Aug. 2008.

[13] A. Sarasa-Renedo, R. Favicchio, U. Birk, G. Zacharakis, C. Malamaki, and J. Ripoll, "Source intensity profile in noncontact optical tomography," Opt. Lett., vol. 35, no. 1, pp. 34-36, Jan. 2010.

[14] L. Carrara, C. Niclass, N. Scheidegger, H. Shea, and E. Charbon, "A gamma, X-ray and high energy proton radiation-tolerant CIS for space applications," in IEEE Solid-State Circuits Conf., Feb. 2009, p. 40

[15] C. Niclass, A. Rochas, A. Besse, and E. Charbon, "Design and characterization of a cmos 3-D image sensor based on single photon avalanche diodes," IEEE J. Solid-State Circuits, vol. 40, no. 9, pp. 1847-1854, Sep. 2005.

[16] M. Hintersteiner, A. Enz, P. Frey, A.-L. Jaton, W. Kinzy, R. Kneuer, U. Neumann, M. Rudin, M. Staufenbiel, M. Stoeckli, K.-H. Wiederhold, and H.-U. Gremlich, "In vivo detection of amyloid- $\beta$ deposits by near-infrared imaging using an oxazine-derivative probe," Nature Biotechnol., vol. 23, no. 5, pp. 577-583, May 2005.

[17] V. Ntziachristos and R. Weissleder, "Experimental three-dimensional fluorescence reconstruction of diffuse media by use of a normalized born approximation," Opt. Lett., vol. 26, no. 12, pp. 893-895, Jun. 2001.

[18] J. Ripoll and V. Ntziachristos, "Imaging scattering media from a distance: theory and applications of noncontact optical tomography," Modern Phys. Lett. B, vol. 18, pp. 1403-1431, May 2004.

[19] A. C. Kak and M. Slaney, Principles of Computerized Tomographic Imaging. Philadelphia, PA: SIAM, 2001.

[20] J. Ripoll and V. Ntziachristos, "From finite to infinite volumes: Removal of boundaries in diffuse wave imaging," Phys. Rev. Lett., vol. 96, p. 173903, May 2006.

[21] M. M. Mohamed and B. F. Sloane, "Cystein cathepsins: Multifunctional enzymes in cancer," Nature Rev. Cancer, vol. 6, pp. 764-775, 2006.

[22] V. Ntziachristos, C.-H. Tung, C. Bremer, and R. Weissleder, "Fluorescence molecular tomography resolves protease activity in vivo," Nature Med., vol. 8, no. 7, pp. 757-760, Jul. 2002.

[23] U. Mahmood, C.-H. Tung, A. Bogdanov, and R. Weissleder, "Near-infrared optical imaging of protease activity for tumor detection," Radiology, vol. 213, pp. 866-870, 1999.

[24] C. Knoess, S. Siegel, A. Smith, and D. Newport, "Performance evaluation of the micropet $\mathrm{r} 4$ pet scanner for rodents," Eur. J. Nucl. Med. Molecular Imag., vol. 30, no. 5, pp. 737-747, 2003.

[25] Y. C. Tai, A. Chatziioannou, S. Siegel, J. Young, D. Newport, R. E. Nutt, and S. R. Cherry, "Performance evaluation of the microPET P4: A PET system dedicated to animal imaging," Phys. Med. Biol., vol. 46, no. 7 , pp. $1845-1862,2001$

[26] C.-H. Tung, U. Mahmood, S. Bredow, and R. Weissleder, "In vivo imaging of proteolytic enzyme activity using a novel molecular reporter," Cancer Res., vol. 60, pp. 4953-4958, Sep. 2000.

[27] M. Rudin, P. M. J. McSheehy, P. R. Allegrini, M. Rausch, D. Baumann, M. Becquet, K. Brecht, J. Brueggen, S. Ferretti, F. Schaeffer, C. Schnella, and J. Wood, "PTK787/ZK222584, a tyrosine kinase inhibitor of vascular endothelial growth factor receptor, reduces uptake of the contrast agent GdDOTA by murine orthotopic B16/BL6 melanoma tumours and inhibits their growth in vivo," NMR Biomed., vol. 18, no. 5, pp. 308-321, Aug. 2005.

[28] P. E. Kinahan, B. H. Hasegawa, and T. Beyer, "X-ray-based attenuation correction for positron emission tomography/computed tomography scanners," Seminars Nucl. Med., vol. 33, no. 3, pp. 166-179, Jul. 2003.

[29] D. Hyde, E. L. Miller, D. H. Brooks, and V. Ntziachristos, "Data specific spatially varying regularization for multimodal fluorescence molecular tomography," IEEE Trans. Med. Imag., vol. 29, no. 2, pp. 365-374, Feb. 2010. 